\title{
Análise da sensibilidade após o uso prévio de dessensibilizantes em clareamento dental
}

\author{
Sensitivity analysis after using desensitizing before to the whitening tooth
}

\section{Camila Lopes Crescente}

Aluna de Graduação do Curso de Odontologia da Universidade São Francisco (USF)

Cristiane Franco Pinto

Professora Doutora do Curso de Odontologia da USF

\section{Resumo}

O objetivo desse estudo foi avaliar a utilização de três diferentes dessensibilizantes dentais antes da aplicação do gel clareador contendo cálcio. Foram selecionados 32 voluntários e estes divididos, aleatoriamente, em 4 grupos ( $n=8)$ : G1) Gel de Peróxido de Hidrogênio a $35 \%$ contendo cálcio $(\mathrm{Gel} \mathrm{PH}+\mathrm{Ca})$; G2) Desensibilize KF 2\% + (Gel PH+Ca); G3) Desensibilize KF 0,2\% + (Gel PH+Ca) e G4) Nano P + (Gel $\mathrm{PH}+\mathrm{Ca}$ ). Os materiais foram aplicados em 3 sessões e, posteriormente, foram realizadas mensurações da sensibilidade utilizando a escala visual analógica de dor, através de estímulos táteis e evaporativos. Não houve diferença estatística $(p>0,05)$ de sensibilidade entre os indivíduos de grupos diferentes, em nenhum dos períodos de avaliação.

Palavras-chave: clareamento dental; sensibilidade da dentina; clareadores; dessensibilizantes dentinários.

\section{AbstRact}

The objective of this study was to evaluate the use of three different dental desensitizing before application of whitening gel with calcium. They selected 32 volunteers and these randomly divided into 4 groups ( $n=8)$ : G1) Hydrogen Peroxide $35 \%$ gel containing calcium ( $\mathrm{Ca}+\mathrm{Gel} P H)$; G2) Desensibilize $\mathrm{KF}$ $+2 \%(\mathrm{Gel} P H+\mathrm{Ca}) ; \mathrm{G} 3)$ Desensibilize $K F+0.2 \%$ $(\mathrm{Gel} P \mathrm{PH}+\mathrm{Ca}$ ) and G4) P+ Nano (Gel PH + Ca). The materials were applied in three sessions were held and after, sensitivity measurements using the visual analogue scale of pain through tactile and evaporative actuations. There was no statistical difference $(p>0.05)$ in sensitivity between individuals of different groups, in any period of assessment.

Keywords: Tooth bleaching; dentin sensitivity; whitening; desensitizing dentin.

\section{Introdução}

$\mathbf{0}$ clareamento dental (CD) é um procedimento estético bastante procurado na Odontologia já que assegura altos índices de satisfação para o tratamento de dentes vitais escurecidos. Trata-se de uma eficiente opção conservadora quando comparado a procedimentos invasivos como a microabrasão, facetas e coroas $(1,2)$. Dentre os seus efeitos colaterais, os mais comuns são: irritação da mucosa e sensibilidade dental moderada (1) e, por isso, sua eficácia tem sido avaliada através de estudos clínicos para análise de cor e também de dor (sensibilidade pós-operatória) (3).

$\mathrm{O}$ produto mais utilizado para o $\mathrm{CD}$ em consultório é o peróxido de hidrogênio nas concentrações de 35 e $40 \%$. Acredita-se que o principal mecanismo de seu efeito clareador está na capacidade do peróxido de difundir-se através da estrutura dental em razão do seu baixo peso molecular e da produção de radicais livres, como o peridroxil (HO2), que tem a função básica de oxidar moléculas mais complexas (cromóforos), quebrando-as em moléculas mais simples que refletem melhor a luz e, consequentemente, proporcionam uma tonalidade mais clara $(2,4)$.

Foram observadas evidências em microscopia eletrônica de varredura (MEV) pertinente às diminuições na microdureza do esmalte, consequência de uma perda mineral, que pode estar relacionada à composição do agente e aos seus subprodutos (5).

Essa perda mineral aliada à penetração do agente clareador nos canalículos dentinários ocasionam a sensibilidade dental, já que o gel estará em contato com as terminações nervosas estabelecidas nestes canalículos (5). A sensibilidade sentida geralmente é reversível e desaparece com o tempo, após o tratamento e/ou então pelo uso de agentes dessensibilizantes. Existem, no mercado, diversos agentes dessensibilizantes com variadas dinâmicas de atuação.

Agentes que possuem na composição fluoretos e nitrato de potássio têm apresentado bons resultados no que diz respeito à redução da sensibilidade pós-operatória (6). Há indicação de seu uso após o tratamento clareador nos casos em que há sensibilidade ou durante o tratamento $(7,8)$. Os fluoretos restringem as respostas nervosas quando ocluem os canalículos dentinários (6). O nitrato de potássio age reduzindo a excitabilidade das fibras nervosas presentes na polpa, através da sua difusão pelos tecidos dentais duros, o que impede a transmissão de sinais dolorosos ao sistema nervoso central (8).

Alguns agentes dessensibilizantes, como o gluconato de cálcio, foram adicionados à fórmula dos géis clareadores, pretendendo reduzir a sensibilidade sem aumentar o tempo de trabalho (8).

O objetivo deste estudo clínico foi avaliar a sensibilidade dental, após a aplicação de dessensibilizantes dentais de diferentes fórmulas e concentrações anteriormente ao tratamento clareador de consultório com géis contendo cálcio na formulação.

\section{Material e Métodos Seleção dos Voluntários}

Após aprovação pelo Comitê de Ética em Pesquisa, da Universidade São Francisco/CAAE: 36079314.6.0000.5514, e atendendo a resolução no 196/96 
do Conselho Nacional de Saúde do Ministério da Saúde, Brasília, DF, 32 voluntários adultos foram selecionados para integrar a população amostral desta pesquisa. Os critérios de inclusão usados foram: 1) apresentar bom estado de saúde geral e oral; 2) interesse em clarear os dentes; 3) ter boa higiene oral; e de exclusão: 1) possuir recessão gengival, 2) demonstrar dor ou desconforto ao estímulo tátil ou evaporativo (baseline); 3) estar em tratamento ortodôntico ou endodôntico; 4) ter utilizado flúor tópico ou dessensibilizantes nos últimos 3 meses; 5) estar sobre tratamento com antibiótico, analgésico ou anti-inflamatório; 6) gestantes ou lactantes; 7) ter dentes manchados por fluorose ou tetraciclina; 8) ser fumante; 9) ter bruxismo ou apertamento dental; 10) ter cárie ou doença periodontal; 11) ter dentes na cor B1. Esses critérios foram utilizados numa triagem realizada pelo menos duas semanas antes do início dos tratamentos. Todas as consultas foram realizadas nas clínicas da Universidade São Francisco - USF - Campus Bragança Paulista. Cada voluntário individualmente foi informado sobre a natureza do estudo, os procedimentos a serem desenvolvidos, riscos, benefícios e a forma de acompanhamento do tratamento. Em caso de concordar com o exposto, assinou um termo de consentimento livre e esclarecido e foi submetido aos exames iniciais. Os voluntários foram examinados clinicamente através de anamnese e de exames extra e intraoral; os pacientes que não se encaixaram nos critérios de inclusão, e/ou se encaixaram nos critérios de exclusão, foram devidamente informados sobre a impossibilidade de sua participação.

\section{Fase Experimental}

Os 32 voluntários foram aleatorizados em 4 grupos experimentais de 8 voluntários cada: G1 (Grupo Controle) - Gel de Peróxido de Hidrogênio a 35\% contendo cálcio (Whiteness HP Blue - FGM, Joinville, Santa Catarina, Brasil); G2 - Desensibilize KF 2\% (FGM, Joinville, Santa Catarina, Brasil) + Gel de Peróxido de Hidrogênio a 35\% contendo cálcio (Whiteness HP Blue - FGM, Joinville, Santa Catarina, Brasil); G3 - Desensibilize KF 0,2\% (FGM, Joinville, Santa Catarina, Brasil) + Gel de Peróxido de Hidrogênio a 35\% contendo cálcio (Whiteness HP Blue - FGM, Joinville, Santa Catarina, Brasil) e G4 - Nano P (FGM, Joinville, Santa Catarina, Brasil) + Gel de Peróxido de Hidrogênio a 35\% contendo cálcio (Whiteness HP Blue - FGM, Joinville, Santa Catarina, Brasil). O experimento foi executado em 14 dias, sendo os tratamentos clareadores realizados no $1^{\circ}, 7^{\circ}$ e $14^{\circ}$ dias. Antes do início dos tratamentos, foram realizadas mensurações da sensibilidade dental de cada voluntário (baseline), sendo que qualquer resposta positiva aos estímulos foi considerada um critério de exclusão da pesquisa, como já citado anteriormente, e, após a aplicação dos dessensibilizantes e dos géis clareadores conforme instruções dos fabricantes (Tabela I) foi realizada a análise de dor.

Tabela I. Aplicação dos materiais segundo instruções do fabricante

\begin{tabular}{|c|c|}
\hline Materiais (Fabricante) & Instruções dos Fabricantes \\
\hline Whiteness HP Blue (FGM, Brasil) & $\begin{array}{l}\text { Ativar o material misturando as duas seringas por } 25 \text { vezes. } \\
\text { Aplicar por } 40 \text { minutos. }\end{array}$ \\
\hline Dessensibilize KF 2\% (FGM, Brasil) & $\begin{array}{l}\text { Após a profilaxia, aplique o gel dessensibilizante sobre os den- } \\
\text { tes. Aguarde por } 10 \text { minutos. Remova o gel com algodão e água } \\
\text { em abundância }\end{array}$ \\
\hline Dessensibilize KF 0,2\% (FGM, Brasil) & $\begin{array}{l}\text { Após a profilaxia, aplique o gel dessensibilizante sobre os den- } \\
\text { tes. Aguarde por } 10 \text { minutos. Remova o gel com algodão e água } \\
\text { em abundância. }\end{array}$ \\
\hline Dessensibilize Nano P (FGM, Brasil) & $\begin{array}{l}\text { Após a profilaxia, o produto deve ser aplicado com o auxílio de } \\
\text { um microaplicador. Friccionar o produto com um disco de feltro } \\
\text { durante } 10 \text { segundos. Deixar o produto em contato com o dente } \\
\text { durante } 5 \text { minutos. Retirar o produto com bolinha de algodão. } \\
\text { Esperar por } 30 \text { minutos para a aplicação do gel clareador. }\end{array}$ \\
\hline
\end{tabular}

\section{Mensuração da Sensibilidade Dental}

Os voluntários receberam instruções verbais e padronizadas quanto à manipulação da escala visual analógica de dor (EVA) (11), que é uma das escalas mais utilizadas para mensurar a dor patológica com objetivos científicos, já que permite minimizar o aspecto subjetivo. Ela consiste em um retângulo de $10,0 \mathrm{~cm}$ de comprimento, com a parte da frente marcada pelas descrições "nenhuma dor" e "dor insuportável" nas extremidades e, na parte de trás, uma régua milimetrada de 10,0 $\mathrm{cm}$ acoplada à escala, sendo esta régua não visível pelo paciente.

Inicialmente, cada paciente recebeu quatro estímulos táteis com o atrito leve de uma sonda exploradora, ocorrendo um intervalo de dois minutos entre cada estímulo, estímulos 1, 2, 3 e 4, respectivamente: no terço médio da face vestibular dos incisivos centrais superior esquerdo (21) e inferior direito (41), e no terço cervical dos primeiros pré-molares superior direito (14) e inferior esquerdo (34). Após dez minutos da aplicação do último estímulo tátil, foram realizados quatro estímulos 
evaporativos nos mesmos dentes (estímulos 5, 6, 7 e 8), consistindo na aplicação de ar da seringa tríplice por 3 segundos, direcionada perpendicularmente à superfície dental e afastada $2,0 \mathrm{~mm}$ do mesmo. O voluntário foi instruído a puxar a parte móvel da escala de acordo com a intensidade do desconforto experimentado a cada estímulo e o avaliador anotou o registro numérico correspondente na parte de trás da escala (3).

\section{Análise Estatística}

As variáveis "Sensibilidade clínica ao estímulo evaporativo" e "Sensibilidade clínica ao estímulo tátil” não apresentaram distribuição normal. Sendo assim, optou-se pela análise não paramétrica destes resultados. Os efeitos principais Grupo e Tempo foram analisados em cada dente. Os quatro grupos foram comparados pelo teste de Kruskal-Wallis dentro de cada dente/tempo. O teste de Friedman foi utilizado para comparar dentro de cada dente/grupo os tempos diferentes. Utilizou-se o programa estatístico BioEstat, versão 5.0, sob o nível de significância de 5\%.

\section{Resultados}

Trinta e quatro pacientes passaram pelo processo de triagem do presente estudo, sendo sete homens e 27 mulheres, com faixa etária entre 18 a 46 anos (média de idade de 22,7 anos), respeitando os critérios de inclusão e exclusão previamente estabelecidos. Destes pacientes, 32 foram selecionados para o experimento (o gráfico 1 representa a caracterização da amostra). Dois voluntários foram excluídos, um durante a pesquisa, porque não compareceu a pelo menos uma das etapas do procedimento, e outro voluntário antes do início do tratamento, porque se encaixou num critério de exclusão (demonstrou dor ou desconforto ao estímulo tátil ou evaporativo).

Não houve diferença estatística ( $p>0,05)$ de sensibilidade a estímulos tátil ou evaporativo entre os indivíduos de grupos diferentes, em nenhum dos períodos de avaliação. O gráfico 2 representa uma análise descritiva dos resultados e discrimina a porcentagem de voluntários de cada grupo que apresentou alguma sensibilidade aos estímulos tátil e evaporativo e aqueles que não sentiram nenhum desconforto frente aos testes de mensuração da sensibilidade.

Gráfico 1. Caracterização amostral

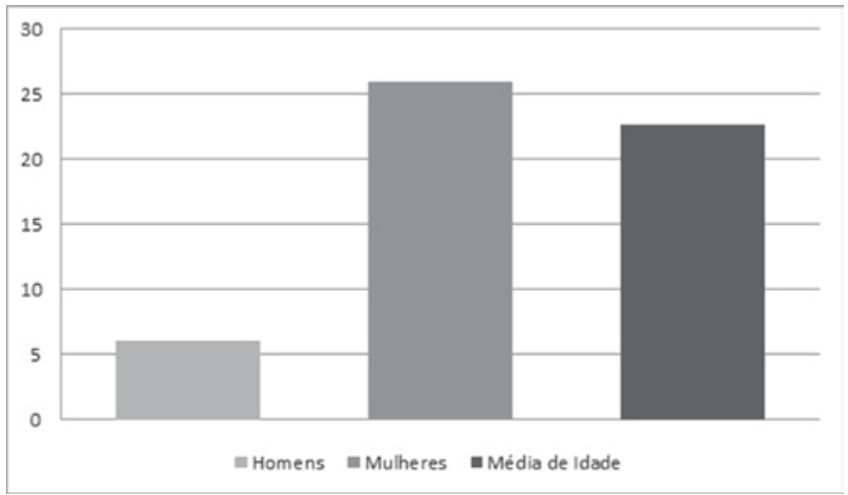

Gráfico 2. Porcentagem de voluntários (\%) que apresentou ou não alguma sensibilidade aos estímulos tátil ou evaporativo para cada agente dessensibilizante

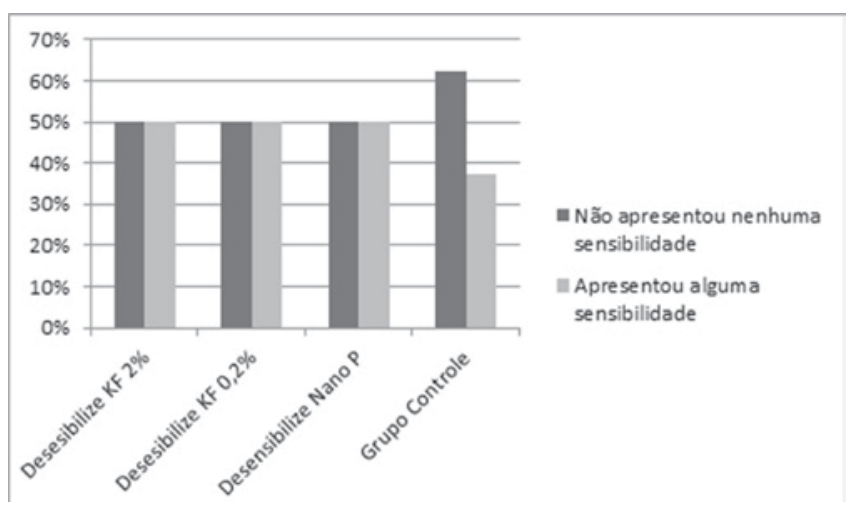

\section{Discussão}

Os resultados deste estudo clínico randomizado demonstraram que a sensibilidade é uma situação de resolução complicada e comum no clareamento de consultório. No presente estudo, 50\% dos voluntários de cada grupo que utilizaram previamente um agente dessensibilizante experimentaram algum tipo de sensibilidade, mas de natureza leve e suportável. Já no grupo controle, apenas 32,5\% dos voluntários relataram algum desconforto. A aplicação dos agentes dessensibilizantes não preveniu a ocorrência de sensibilidade quando comparado ao grupo controle.

Os resultados desta pesquisa equiparam-se às conclusões de BRITO (3), que constatou que o fluoreto de sódio usado nas concentrações de $0,11 \%$ e $2 \%$ não foi capaz de reduzir a sensibilidade dentinária.

No entanto, diferenciam-se daqueles encontrados por AJCHARANUKUL et al. (9), que observaram, através de um estudo realizado em humanos, que o uso de substâncias à base de potássio reduziu a hipersensibilidade dentinária de lesões cervicais e eliminou a resposta dolorosa em 11 de 14 dentes testados.

A etiologia da sensibilidade provocada pelo clareamento é complexa, contudo a Teoria Hidrodinâmica de Brannstrom (10) é a mais aceita para explicar tal evento e afirma que a sensibilidade dental seria causada pela movimentação do peróxido de hidrogênio, através do esmalte e da dentina (movimento de fluido nos túbulos dentinários). Esse movimento ativaria os nociceptores, que inervam tais túbulos, e resultaria na percepção de dor: sensibilidade. Segundo BRANNSTROM (10), calor, frio e pressão poderiam causar uma movimentação mais rápida do fluido nos túbulos e ocasionar um aumento na 
sensibilidade, por isso esperava-se que os voluntários apresentassem uma maior sensação de dor aos estímulos de frio, calor ou pressão (testes de mensuração da sensibilidade utilizados neste estudo).

Um tratamento sugerido para a redução da sensibilidade dental é o uso de agentes dessensibilizantes à base de fluoretos, anterior ou posteriormente ao tratamento clareador. Os fluoretos agem na obliteração dos canalículos dentinários abertos $(6,11)$, eliminando as respostas nervosas ao impedir o contato de fluidos com a câmara pulpar $(12,13,14)$, o que bloqueia o mecanismo hidrodinâmico descrito por BRANNSTROM (10).

Uma das hipóteses que pode explicar a ausência de efeito dessensibilizante dos agentes à base de fluoretos em, aproximadamente, $50 \%$ da amostra é que o tempo para deposição de compostos fluoretados na superfície dental pode não ter sido suficiente para obliterar túbulos e amenizar a movimentação de fluidos em seu interior. Segundo MARINI et al. (15), o flúor em pequenas e constantes concentrações é capaz de reduzir a sensibilidade dentinária apenas a partir da quarta semana de uso. Esse fato pode ficar ainda mais complicado quando somente o esmalte é exposto, ou seja, neste estudo não existiu dentina exposta, o contato direto ocorreu entre o gel clareador e/ou o gel de flúor e a superfície de esmalte.

JOSEY et al. (16) observaram evidências em microscopia eletrônica de varredura (MEV) quanto à dissolução e aumento de porosidade da superfície do esmalte tratado com gel clareador e CAVALLI et al. (5) constataram uma diminuição dos valores de microdureza do esmalte clareado, devido à perda mineral, ambos os fatos determinam a sensibilidade dental pós operatória e podem ter sido agentes causadores dos casos de sensibilidade apresentados.

O efeito dessensibilizante dos fluoretos e nitrato de potássio é uma hipótese confirmada também por KOSE (17), que constatou uma diminuição na intensidade da sensibilidade causada por clareamento caseiro (peróxido de carbamida a 16\%), quando utilizou um agente dessensibilizante à base de tais componentes previamente ao CD.

A alta concentração do gel clareador usado poderia ter colaborado com o índice de pacientes com sensibilidade, durante e após o tratamento clareador, pois, se analisarmos criteriosamente estudos clínicos nos quais os pesquisadores utilizaram similarmente o gel de Peróxido de Hidrogênio a 35\%, durante o clareamento de consultório, observar-se-á que os resultados demonstram uma variação entre 67 e $87 \%$ no índice de pacientes com sensibilidade dental $(8,18)$, no entanto, frente aos nossos resultados observou-se que apenas $32,5 \%$ dos pacientes do grupo 1 (que usaram apenas o gel clareador) sentiram algum tipo de sensibilidade.

O evento descrito anteriormente pode ser explicado pela presença do cálcio na composição do gel clareador utilizado (Whiteness HP Blue 35\% - FGM, Joinville, Santa Catarina, Brasil). Esperava-se que a sensibilidade seria minimizada já que, segundo seus fabricantes, o objetivo da incorporação do gluconato de cálcio à composição destes géis foi prevenir a desmineralização do esmalte dental durante o procedimento de clareamento e reduzir a sensibilidade dental. Através dos resultados de análise de microdureza feitos por CAVALLI et al. (5), que comparou amostras de dente humano clareadas com gel clareador puro e outros dois associados a flúor e cálcio, foi constatada a redução da perda de microdureza naqueles em que utilizou-se gel associado a flúor e cálcio.

Diante do exposto sugere-se a realização de futuras pesquisas envolvendo os agentes redutores da sensibilidade dental.

\section{Conclusão}

O uso dos agentes dessensibilizantes previamente ao clareamento dental de consultório com Peróxido de Hidrogênio a 35\% contendo cálcio não foi capaz de reduzir significativamente a sensibilidade quando comparado ao grupo controle. 


\section{Referências ::}

1. Haywood VB, Heymann HO. Nightguard vital bleaching. Quintessence Int. 1989;20:173-6.

2. Araujo FDE, Baratieri LN, Araujo E. In situ study of in-office bleaching procedures using light sources on human enamel microhardness. Oper Dent. 2010;35(2):139-46.

3. Brito MML. Avaliação clínica do efeito do fluoreto de sódio sobre a sensibilidade dentinária, a saúde gengival e a satisfação do paciente durante o clareamento dental caseiro com peróxido de carbamida a $10 \%$. Tese de Doutorado, Faculdade de Odontologia de Piracicaba- UNICAMP, 2008.

4. Haywood VB. Nightguard vital bleaching: curent concepts and research. J Am Dent Assoc. 1997;128(suppl):19-25.

5. Cavalli V, Reis AF, Giannini M, Ambrosano GMB. The effect of elapsed time following bleaching on enamel bond strength of resin composite. Oper Dent. 2001;26:597-602.

6. Tay LY, Kose C, Loguercio AD, Reis A. Assessing the effect of a desensitizing agent used before in-offie tooth bleaching. J Am Dent Assoc. 2009;140(10):1245-51.

7. Attin T, Betke H, Schippan F, Wiegand A. Potencial of fluoridated carbamide peroxide gels to support post-bleaching enamel re-hardening. Journal of Dentistry. 2007;35:755-9.

8. Matis BA, Cochran MA, Eckert G. In vivo study of two carbamide peroxide gels with different desensitizing agents. Operative Dentistry. 2007;32(6):549-55.

9. Ajcharanukul O, Kraivaphan P, Wanachantararak S, Vongsavan N, Matthews B. Effects of potassium ions on dentine sensitivity en man. Arch Oral Biol. 2007;52:632-9.
10. Brannstrom, M. Dentin sensitivity. Arsb Goteb Tandlak Sallsk. 1964:15-35.

11. Haywood VB, Caughman F, Frazier KB, Myers ML. Tray delivery of potassium nitrate-fluoride to reduce bleaching sensitivity. Quintessence Int. 2001;32:105-9.

12. Tam, L. Effect of potassium nitrate and fluoride on carbamide peroxide bleaching. Quintessence Int. 2001;32(10):766-70.

13. Cummins, D. The Efficacy of a New Dentifrice Containing $8.0 \%$ Arginine, Calcium Carbonate, and 1450 ppm Fluoride in Delivering Instant and Lasting Relief of Dentin Hypersensitivity. J Clin Dent. 2009;20(4):109-14.

14. Peixoto LM, Daleprane B, Battitucci MHG, Sanglard L, Pazinatto FB. Tratamento da hipersensibilidade dentinária cervical. Rev. Bras. de pesq. em saúde. 2010;12(2):69-74.

15. Marini I, Checchi L, Vecchiet F, Spiazzi L. Intraoral fluoride releasing device: a new clinical therapy for dentine sensitivity. J Periodontol. 2000;71(1):90-5.

16. Josey AL, Meyers IA, Romaniuk K, Symons AL. The effect of a vital bleaching technique on enamel surface morphology and the bonding of composite resin to enamel. J Oral Rehab. 1996;23:244-50.

17. Kose CJ. Avaliação clínica de um gel dessensibilizante na prevenção da sensibilidade decorrente do clareamento caseiro. Dissertação de Mestrado, Universidade estadual de Ponta Grossa. Ponta Grossa, 2010. 54p.

18. Reis A, Dalanhol AP, Cunha TS, Kossatz S, Loguercio AD. Assessment of tooth sensitivity using a desensitizer before light-activated bleaching. Oper Dent. 2011;36(1):12-7.

Recebido em: 22/09/2015 / Aprovado em: 28/10/2015

Camila Lopes Crescente

Rua 22 de julho, 311, Centro

Extrema/MG, Brasil - CEP: 37640-000

E-mail: micrescente@hotmail.com 